\title{
Markers of Proliferative Activity Are Predictors of Patient Outcome for Low-Grade Endometrioid Adenocarcinoma But Not Papillary Serous Carcinoma of Endometrium
}

\author{
Abdulmohsen Al Kushi, M.D., Peter Lim, M.D., Christina Aquino-Parsons, M.D., C. Blake Gilks, M.D. \\ Departments of Pathology (AA K, CBG) and Radiation Oncology (PL, CA-P), Vancouver General Hospital \\ and British Columbia Cancer Agency, Vancouver, British Columbia, Canada
}

On the basis of pathogenesis, two types of endometrial cancer can be recognized. Type 1 endometrial carcinomas are relatively indolent tumors that develop after prolonged estrogen stimulation, on a background of endometrial hyperplasia. Type 2 endometrial carcinomas are aggressive tumors that are not associated with hyperplasia or estrogen $\mathrm{ex}$ cess. The aim of this study is to evaluate the prognostic significance of tumor proliferative activity in early-stage endometrial cancer by using mitotic index and immunostaining, comparing Type 1 (endometrioid) and Type 2 (papillary serous carcinoma) tumors. The mitotic index, MIB-1, and p53 immunostaining in 39 tumors from patients with lowgrade Stage Ia or Ib endometrioid adenocarcinoma; as well as 23 tumors from patients with Stage I papillary serous carcinoma. In low-grade endometrioid adenocarcinoma, mitotic and MIB-1 indices were statistically significant independent prognostic indicators $(P=.004$ and $P=.018$, respectively), and both were strongly correlated with p53 expression $(P=.01$ and $P=.006$, respectively). The mean mitotic index was 5 mitoses/10 high-power fields, and mean MIB-1 index was 27.5\%. There was no significant correlation between mitotic or MIB-1 indices and patient outcome or p53 expression in papillary serous carcinoma. The mean mitotic index was 31 mitoses/10 high-power fields, and mean MIB-1 index was $30.5 \%$ in these tumors. p53 expression and proliferative indices are strongly correlated in low-grade endometrioid adenocarcinoma. MIB-1 and mitotic indices are inde-

Copyright (C) 2002 by The United States and Canadian Academy of Pathology, Inc

VOL. 15, NO. 4, P. 365, 2002 Printed in the U.S.A.

Date of acceptance: December 20, 2001

The results of this study were presented, in part, at the USCAP Annual Meeting, Atlanta, Georgia, March 3-9 2001.

Address reprint requests to: C.B. Gilks, M.D., Department of Pathology, 910 W. 10th Avenue, 1st floor, Vancouver Hospital and Health Science Centre, Vancouver, BC V5Z 4E3, Canada; fax: 604-875-5707; e-mail: bgilks@vanhosp.bc.ca. pendent prognostic indicators in these tumors. Papillary serous carcinoma of endometrium is rapidly proliferative in tumors even at an early stage, and quantification of proliferative activity in these tumors does not allow prediction of patient outcome.

KEY WORDS: Endometrial carcinoma, MIB-1, Mitotic index, p53, Papillary serous carcinoma, Proliferative activity.

Mod Pathol 2002;15(4):365-371

Endometrial cancer is the fourth most common malignancy in North American women. In the United States, there are 33,000 newly diagnosed cases and 4,000 deaths per year $(1,2)$. On the basis of the proposed dualistic model of endometrial carcinogenesis, there are two types of endometrial carcinoma that differ with regard to epidemiologic risk factors, histopathologic lesions, and molecular events (3). Type 1 endometrial carcinomas are relatively indolent tumors that develop after prolonged estrogenic stimulation. Low-grade endometrioid adenocarcinoma is the prototype of Type 1 endometrial carcinoma. Although most of these patients do well, recurrence and death due to disease can occur. Type 2 endometrial carcinomas are more aggressive tumors that are not associated with endometrial hyperplasia or estrogen excess. These tumors develop de novo in the atrophic endometrium of older postmenopausal women (4-7).

Papillary serous carcinoma of endometrium (PSCE) is the prototype of Type 2 endometrial carcinoma and is an uncommon aggressive variant of endometrial cancer. It accounts for $1-10 \%$ of cases of endometrial carcinoma and for a disproportionately high percentage of patients who die of endometrial cancer (8-12).

p53 mutation and its overexpression are more common in PSCE than in endometrioid adenocarcinoma. p53 overexpression as assessed by immunostaining is seen in $\leq 85 \%$ of PSCE tumors, compared 
with in $20 \%$ of endometrioid adenocarcinomas, and correlates with mutation of the p53 gene (13-18). We have previously reported that p53 overexpression is associated with a poor outcome for patients with lowrisk endometrial carcinoma (defined at our center as Stage Ia or Ib, Grade 1 or 2 endometrioid endometrial adenocarcinoma, without vascular invasion; 19). p53 overexpression was previously shown to be correlated with advanced PSCE stage (20).

Our aims were to (1) evaluate the prognostic value of assessing proliferative activity by using mitotic and MIB-1 indices in early-stage endometrial carcinoma, (2) study the correlation between these indices and p53 overexpression, and (3) attempt to identify a subset of low-grade endometrioid adenocarcinoma tumors with high proliferative indices and p53 overexpression that behave similarly to Type 2 endometrial carcinoma, or a subset of PSCE tumors with low proliferative indices and p53 negative immunostaining, with a relatively favorable outcome.

\section{MATERIAL AND METHODS}

\section{Cases Selection}

The British Columbia Cancer Agency-Vancouver is a regionally based comprehensive cancer center with a catchment population of approximately 2.2 million people. Cases were selected as follows:

For low-grade endometrioid adenocarcinoma: between January 1992 and August 1996, 406 patients with endometrioid endometrial adenocarcinoma limited to the uterine corpus with $<50 \%$ myometrial invasion, no vascular invasion, and no Grade 3 disease were identified. Grading was done according to the 1988 revision of the International Federation of Gynecology and Obstetrics system (FIGO-88). Tumors with any component of papillary serous or clear-cell differentiation were excluded. Of these tumors, 315 were found to be diploid by flow cytometry. These latter patients were considered to have low-risk endometrial carcinoma and received no further treatment. The 5-year disease-specific survival for these patients was $95 \%$. Fourteen patients in the low-risk group developed recurrent disease. Eleven of the recurrent tumors and 28 stage- and grade-matched controls chosen from this cohort of patients were selected for this study.

For papillary serous carcinoma: between 1985 and 1995, 64 patients with papillary serous carcinoma of endometrium limited to the uterine corpus (Stage I) were identified, and staging was done according to the FIGO-88 system. The 5-year diseasespecific survival was $75 \%$. Formalin-fixed paraffinblocks and slides were available for 23 cases. Clinical follow-up information from 4-144 months (mean, $58 \mathrm{mo}$ ) was available for these patients. Six of these patients died of the disease, and 17 were alive with no disease during the follow-up period. Treatment of these patients was variable, although we observed a significantly better outcome for patients receiving postoperative whole-abdominal radiotherapy (21).

\section{Pathological Features}

\section{and Immunohistochemistry}

The hematoxylin and eosin (H\&E) slides for all cases selected were reviewed; the proliferative activity of these tumors was evaluated using mitotic and MIB-1 indices. The mitotic index was done on $\mathrm{H} \& \mathrm{E}$ sections by counting the number of mitoses in five sets of 10 randomly selected high-power fields (10HPF; $400 \times$ ) of the tumor, and the highest count/ $10 \mathrm{HPF}$ was used. One paraffin block containing representative portions of the tumor was selected from each case for MIB-1 and p53 immunostaining. The avidin-biotin (ABC) method was used for immunostaining and applied to formalin-fixed and paraffin-embedded tissue. Serial sections were cut at $3 \mu \mathrm{m}$, deparaffinized with xylene, and rehydrated through a series of graded alcohols. The immunostaining procedure was performed using an automated stainer (Ventana, Tucson, AZ). The primary antibodies were used against Ki-67 protein (MIB-1, Immunotech, 1:200) and p53 (DO-7, DAKO, 1:400). The anti-p53 antibody DO-7 recognizes both wildtype and mutant p53. In contrast to wild-type p53, which is relatively unstable and expressed in small amounts, mutant p53 is overexpressed and therefore remains longer in the nucleus. Sections were counterstained with light hematoxylin. p53 nuclear staining of $<5 \%$ of tumor nuclei was considered negative, staining of $\geq 5-50 \%$ of tumor nuclei was considered weak positive, and staining of $>50 \%$ of tumor nuclei was considered strong positive.

MIB-1 index was assessed by counting 500 tumor cell nuclei in at least five fields under a grid at $400 \times$ magnification ( $10 \times$ ocular and $40 \times$ objective) and calculating the percentage of positive nuclei. All degrees of nuclear staining intensity were considered positive.

\section{Statistical Analysis}

Mann-Whitney $U$ test was used for comparison of median mitotic and MIB-1 indices with patient outcome for both low-risk endometrial carcinoma and PSCE. Fisher's exact test was used to analyze a $2 \times$ 2 table comparing the subset of low-grade endometrioid carcinoma patients with high proliferative indices and p53 overexpression with the rest of the patients with regard to recurrence rate. The same test was also used to analyze a $2 \times 2$ table compar- 
ing the subset of PSCE patients with low proliferative indices and p53 negativity with the rest of the patients with regard to patient outcome. Scatterplots were used to assess the linear association and simple linear regression analysis between mitotic and MIB-1 indices in both tumors. Spearman's rank correlation coefficient was used as an estimator of the linear correlation. Calculations and production of graphs were done using SPSS software, Version 9.0 (SPSS, Chicago, IL).

\section{RESULTS}

Thirty-nine low-grade endometrioid adenocarcinomas were studied, 11 from patients who experienced recurrence on follow-up and 28 stage- and grade-matched controls who did not have a documented recurrence in the same follow-up period. The mean mitotic index of these tumors was 5 mitoses/10HPF (median was 3 mitoses/10HPF), and $>65 \%$ of the tumors had $<6$ mitoses/10HPF (Fig. 1). The mean MIB-1 index of these tumors was $27.5 \%$ (median was $27 \%$ ). These markers of proliferative activity were found to be independent predictors of the patient outcome in low-grade endometrioid adenocarcinomas, with $\mathrm{P}=.004$ in the case of mitotic index and $\mathrm{P}=.018$ in the case of MIB-1 index (Figs. 2-4). There is strong positive correlation between mitotic and MIB-1 indices with a Spearman's rank correlation coefficient $\left(r_{s}\right)$ of 0.85 (Fig. 5). These proliferative indices were significantly correlated with p53 overexpression status in low-risk endometrial carcinomas with $\mathrm{P}=.01$ in case of mitotic index versus p53 and $\mathrm{P}=.006$ in the case of MIB-1 index versus p53 (Fig. 6).

We analyzed 23 Stage I papillary serous carcinomas of endometrium. Six of these patients died of disease during the follow-up period, and 17 were

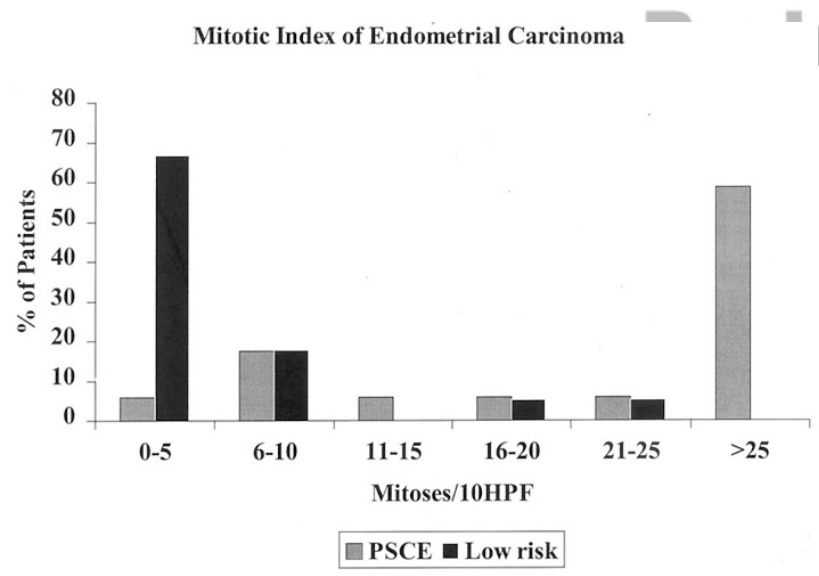

FIGURE 1. Mitotic index in low-risk endometrial carcinoma and papillary serous carcinoma of endometrium (PSCE) tumors. More than $65 \%$ of low-risk tumors have a mitotic index of $<6$ mitoses/10HPF, compared with $>55 \%$ of PSCE tumors that have a mitotic index of $>25$ mitoses/10HPF. alive with no disease. The mean mitotic index of these tumors was 31 mitoses/10HPF (median was 28 mitoses/10HPF), and $>55 \%$ of the tumors had $>25$ mitoses/10HPF (Fig. 1). The mean MIB-1 index of these tumors was $30.5 \%$ (median was $31.0 \%$ ). We did not find these proliferative indices of prognostic significance in Stage I PSCE (Figs. 2-3). The relationship between mitotic and MIB-1 indices in these tumors showed a weak positive correlation with a Spearman's rank correlation coefficient $\left(\mathrm{r}_{\mathrm{s}}\right)$ of 0.62 (Fig. 5). These proliferative indices were also not significantly correlated with p53 overexpression status in PSCE tumors (Fig. 6).

Low-grade endometrioid adenocarcinoma with high proliferative indices (defined as $>5$ mitoses/ 10HPF) and p53 overexpression were compared with the rest of the group of low-grade endometrioid carcinoma (Table 1). There were relatively few patients with high proliferative index and p53positive tumors. The outcome for patients whose tumors showed both increased mitotic activity and p53 overexpression was worse than that of patients whose tumors lacked these features, but this difference was not statistically significant $(P=.083)$. No difference in outcome was observed when papillary serous carcinomas with low proliferative indices and p53 negativity were compared with those papillary serous carcinomas lacking these features (data not shown).

\section{DISCUSSION}

Endometrial adenocarcinoma has been subdivided into two distinct clinicopathological groups that differ not only with respect to their histology but also with respect to risk factors, natural history, outcome, and molecular events during tumorigenesis. In this study, we looked at these two groups of

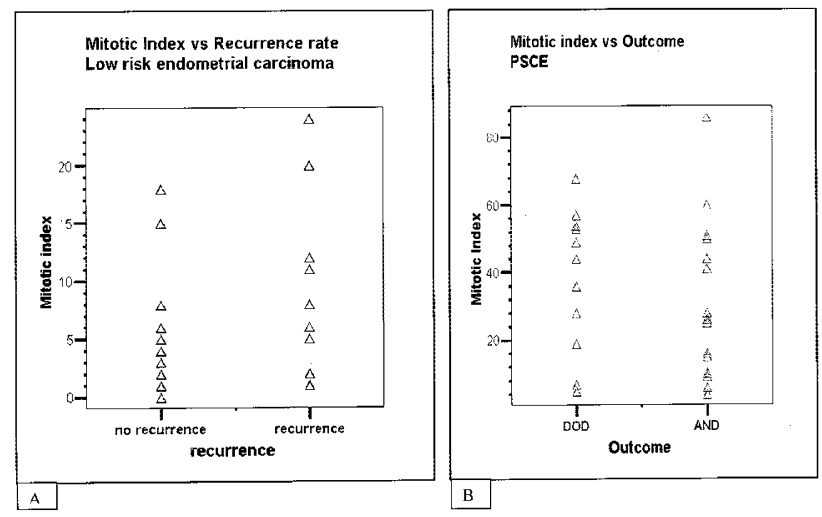

FIGURE 2. Scatterplots showing the relationship between patient outcome and mitotic index of the tumor (A) in low-risk endometrial carcinoma; $\mathrm{P}=.004,(\mathrm{~B})$ papillary serous carcinoma; $P$ value is not significant. DOD, dead of disease; AND, alive—no disease. 

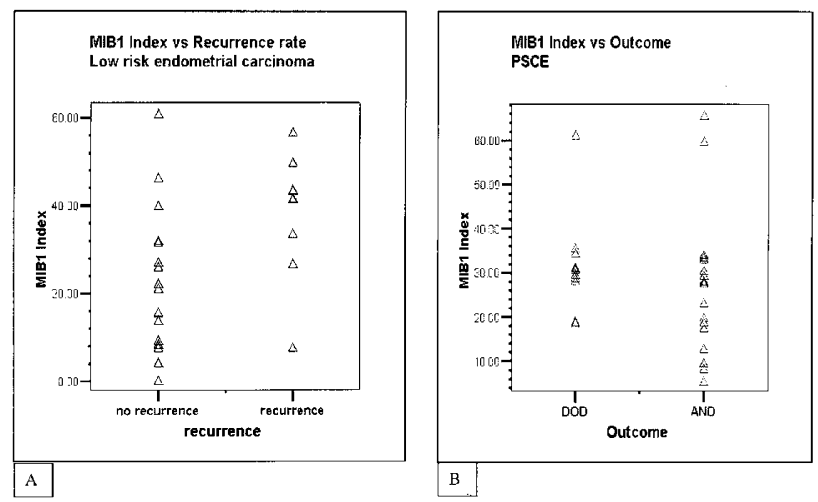

FIGURE 3. Scatterplots showing the relationship between patient outcome and MIB-1 index of the tumor (A) in low-risk endometrial carcinoma; $\mathrm{P}=.018$, (B) papillary serous carcinoma; $P$ value is not significant. DOD, dead of disease; AND, alive—no disease.

patients with endometrial carcinoma to evaluate proliferative activity in their tumors.

The proliferative activity of a tumor has long been considered to bear a relationship to its clinical course, and recent reports indicate that measurement of tumor cell proliferation yields useful prognostic information. There are several methods to measure cell proliferation. These include counting mitotic figures, S-phase fraction assessed by ${ }^{3} \mathrm{H}-$ thymidine uptake or by DNA flow cytometry, or immunostaining for the proliferating cell nuclear antigen or MIB-1/Ki-67 index. MIB-1 is a monoclonal antibody that recognizes a nuclear antigen that is expressed in all cell cycle phases except $\mathrm{G}_{0}$. MIB-1 monoclonal antibody is a reliable means of assessing the growth fraction of normal tissues and has been used to study the cell proliferation in various cancers (22-34).

Salvesen et al. (35) found MIB-1 immunostain performed on paraffin-embedded tumor tissue to be an independent prognostic indicator of survival in 142 patients with endometrial carcinoma. In another study series by Geisler et al. (36), 147 consecutive patients with endometrial carcinoma who had no systemic therapy were followed for $\geq 60$ months, and the authors showed that MIB-1 index was an independent prognostic indicator of 5-year survival, particularly in patients with Stage I disease. Geisler et al. (37), in an earlier study on a series of 39 patients, had shown MIB-1 to be indicator of increased risk of recurrence. Nordstrom et al. (38) found MIB-1/Ki-67 expression and S-phase fraction were significantly related to disease-specific survival in patients with endometrial carcinoma. Despite the methodological differences between these studies, all found MIB-1 to be an independent prognostic indicator of survival. Our study differs from these earlier studies in separately considering lowgrade, low-risk tumors and high-grade papillary serous tumors. As expected, the latter group had a

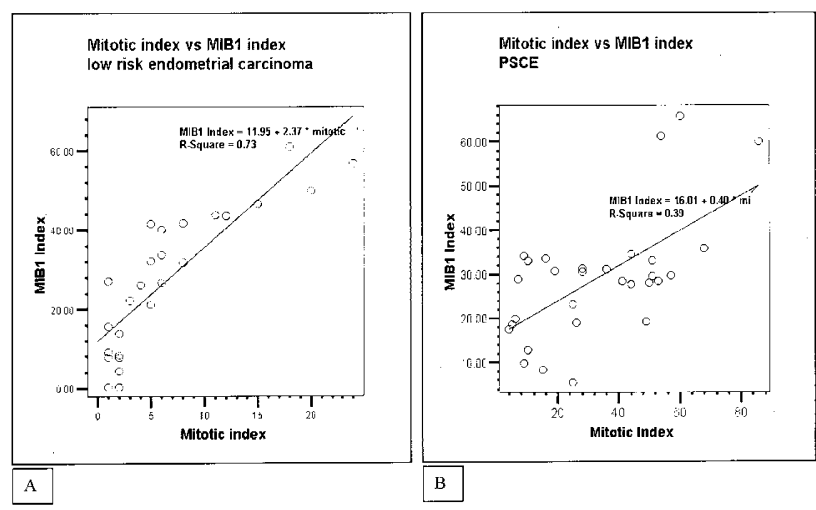

FIGURE 4. Simple linear regression plots of mitotic and MIB-1 indices in (A) low-risk endometrial carcinoma; $r \mathrm{~s}=0.85$, (B) papillary serous carcinoma of endometrium; $r \mathrm{~s}=0.62$.

much higher proliferative rate in our study. We found that the mitotic index, which is relatively simple and applicable in routine pathology practice, is also an independent prognostic factor and is strongly correlated with MIB-1 index in low-grade endometrioid carcinoma.

In PSCE, MIB-1 or mitotic index did not correlate with outcome, and the correlation between mitotic and MIB-1 indices in these tumors was weak. Although expression of $\mathrm{Ki}-67$ is consistently seen in normal proliferating cells, it may be lost in highgrade tumor cells. Mitotic index is simpler than immunostaining, and on the basis of these results, it is a better measure of cell proliferation than is MIB-1 staining in these tumors.
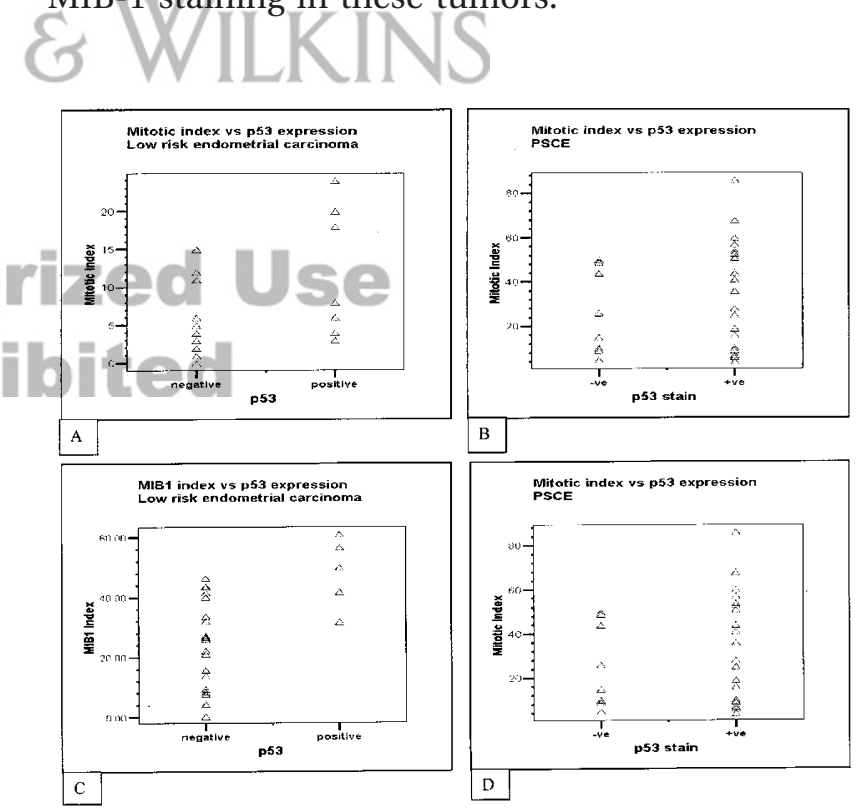

FIGURE 5. Scatterplots showing the relationship between proliferative indices and p53 overexpression in both tumors. A, mitotic index and p53 overexpression in low-risk endometrial carcinoma; $\mathrm{P}=$ .01. B, mitotic index and p53 overexpression in papillary serous carcinoma of endometrium (PSCE); $P$ value is not significant. C, MIB-1 index and p53 overexpression in low-risk endometrial carcinoma; $\mathrm{P}=$ .006. D, MIB-1 index and p53 overexpression in PSCE; $P$ value is not significant. 
Disease Free Survival of Low Grade Endometrioid Adenocarcinoma

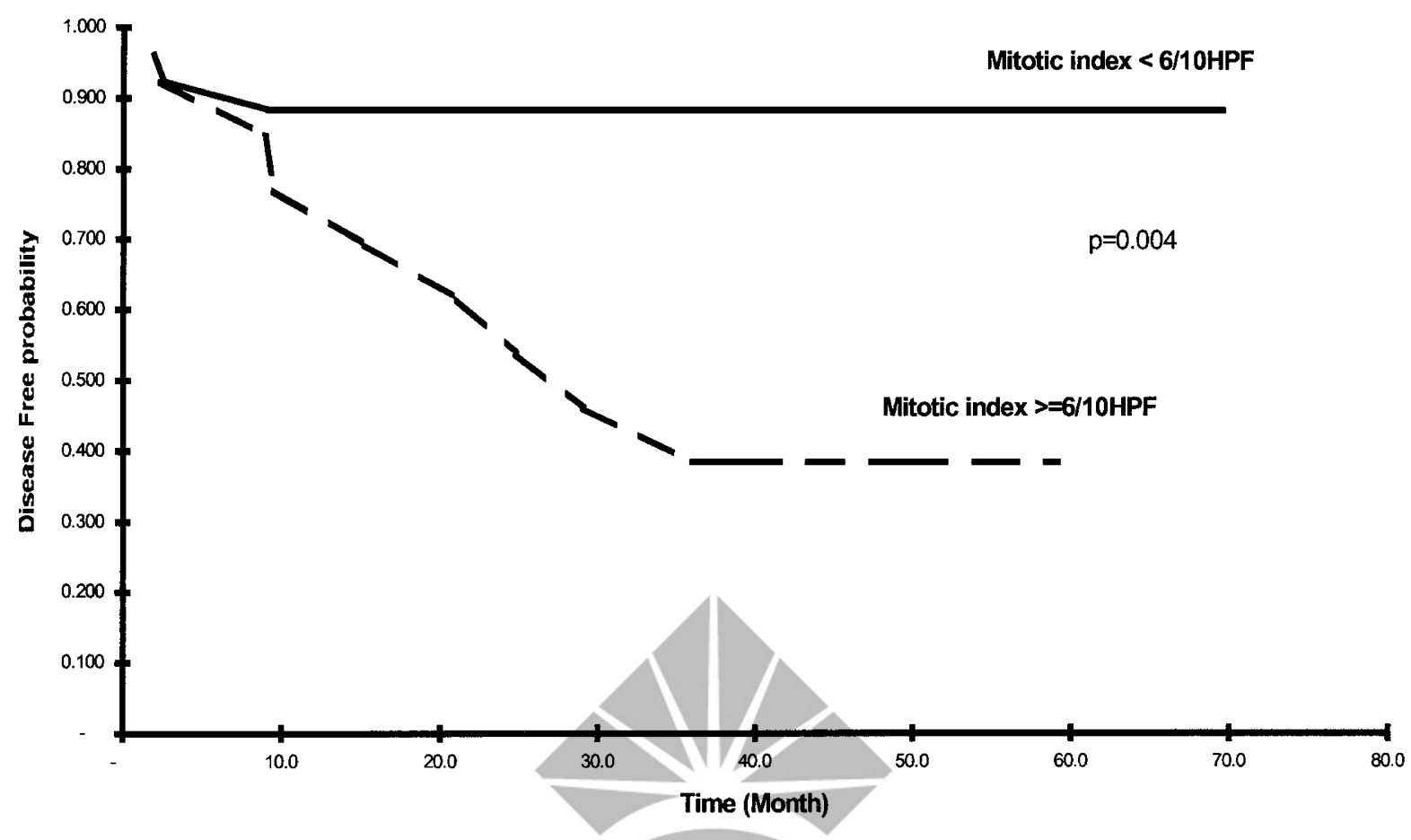

FIGURE 6. The disease-free probability for the low-grade endometrioid adenocarcinoma patients, comparing tumors with $<6$ mitoses/10HPF (upper curve) and tumors with $\geq 6$ mitoses/10HPF (lower curve). $\mathrm{P}=.004$.

TABLE 1. p53 Overexpression and Mitotic Index in LowGrade Endometrioid Endometrial Adenocarcinoma

\begin{tabular}{lccc}
\hline \multicolumn{1}{c}{ Group } & No Recurrence & Recurrence & Total \\
\hline Rest of patients & 25 & 7 & 32 \\
P53 positive and $\mathrm{MI} \geq 6 /$ & 3 & 4 & 7 \\
Total & 28 & 11 & 39 \\
\hline
\end{tabular}

MI, mitotic index (mitotic figures/10 high-power fields).

Mitotic index is used routinely in the grading of breast carcinoma and increasingly is applied to the grading of ovarian carcinoma $(39,40)$. Pirog and Czerwinski (41) have shown that a significantly higher 5-year mortality rate was associated with a mitotic index of $>5$ mitoses/10HPF in endometrial adenocarcinoma. Tornos et al. (42) identified $\geq 8$ mitoses/10HPF as a statistically significant adverse prognostic factor in Stage I, Grade I endometrioid endometrial adenocarcinoma. FIGO grading of uterine endometrial endometrioid carcinoma requires evaluation of histologic features, including recognition of the amounts of solid growth, distinction of squamous from nonsquamous solid growth, and assessment of degree of nuclear atypia (43). It does not include mitotic index. Assessment of mitotic index could easily be incorporated into the grading of endometrial carcinoma, as is done for breast and ovarian carcinoma, and this potentially could improve our prognostication for patients with these tumors.
Low-grade tumors tend to have a mitotic index of $<10$ mitoses/10HPF, and high-grade tumors, $>10$ mitoses/10HPF. Our results suggest that endometrial carcinoma can be divided into three groups based on the mitotic index, with low, intermediate, and high risk of recurrence, using the cutoffs of $\leq 5$, $6-10$, and $>10 / 10 \mathrm{HPF}$, respectively. It is possible that a grading system combining architecture, nuclear grade, and mitotic index could replace the current practice of subclassifying endometrial cancer based on cell type and, in the case of endometrioid carcinoma, tumor architecture. This possibility would have to be tested in a large series of patients with endometrial adenocarcinoma.

p53 overexpression is more frequently seen in papillary serous carcinoma than in endometrioid adenocarcinoma (13). We have shown in previous studies that p53 overexpression can be of prognostic significance in low-risk endometrial carcinoma but not in papillary serous carcinomal $(9,21)$. p53 overexpression in endometrial carcinoma was correlated with higher cell proliferative indices, such as MIB-1 index, as shown by Ioffe et al. (44). In this study, we looked at the correlation between p53 overexpression and proliferative activity markers. We found significant correlation between both markers of proliferative activity (mitotic and MIB-1 indices) and p53 in low-risk endometrial carcinoma $(\mathrm{P}=.001$ and $\mathrm{P}=.006$, respectively) but not in PSCE. 
We hypothesized that a subset of tumors classified as low-grade/low-risk by conventional histopathological assessment would show immunophenotypic and cell proliferation features of highgrade tumors. Accordingly, we compared low-grade endometrioid carcinoma with increased mitotic index and p53 overexpression to tumors lacking these features. The difference in outcome for these patients approached but did not reach statistical significance $(\mathrm{P}=.08)$ when we used the combination of mitotic index of $>5 / 10 \mathrm{HPF}$ and p53 overexpression to define this subset of patients. A similar approach was also used for the patients with PSCE. In this group of patients, we tried to recognize a subset with low proliferative markers and lacking p53 overexpression whose tumors behave less aggressively than those of other PSCE patients. Separation of such a subset from the rest of PSCE patients did not prove possible.

In summary, markers of proliferative activity, particularly mitotic index, are good predictors of patient outcome in low-grade endometrioid adenocarcinoma. Routine inclusion of mitotic index in assessment of tumor grade could improve prognostication of these tumors.

\section{REFERENCES}

1. Rose PG. Endometrial carcinoma. N Engl J Med 1996;335: $640-9$.

2. Kurman RJ, Zaino RJ, Norris HJ. Endometrial carcinoma. In Kurman R, editor. Blaustein's pathology of the female geñital tract. 4th ed. New York: Springer-Verlag; 1994, p. 439-86.

3. Lax SF, Kurman RJ. A dualistic model for endometrial carcinogenesis based on immunohistochemical and molecular genetic analyses. Verh Dtsch Ges Pathol 1997;81:228-32.

4. Burton JL, Wells M. Recent advances in the histopathology and molecular pathology of carcinoma of the endometrium. Histopathology 1998;33:297-303.

5. Sherman ME. Theories of endometrial carcinogenesis: a multidisciplinary approach. Mod Pathol 2000;13:295-308.

6. Ellenson LH. The molecular biology of endometrial tumorigenesis: does it have a message? Int J Gynecol Pathol 2000; 19:310-3.

7. Koshiyama M, Konishi I, Fujii S. Pathology, hormonal aspects, and molecular genetics of the two types of emdometrial cancer. Cancer J 1998;11:277-83.

8. Grice J, Ek M, Greer B, Koh WJ, Muntz HG, Cain J, et al. Uterine papillary serous carcinoma: evaluation of long-term survival in surgically staged patients. Gynecol Oncol 1998; 69:69-73.

9. Hendrickson MR, Ross J, Eifel P, Martinez A, Kempson RL. Uterine papillary serous carcinoma: a highly malignant form of endometrial adenocarcinoma. Am J Surg Pathol 1982;6: 93-108.

10. Sherman ME, Bitterman P, Rosenshein NB, Delgado G, Kurman RJ. Uterine serous carcinoma: a morphologically diverse neoplasm with unifying clinicopathologic features. Am J Surg Pathol 1992;16:600-10.

11. Goff BA, Kato D, Schmidt RA, Ek M, Muntz HG, Cain JM, et al. Uterine papillary serous carcinoma: patterns of metastatic spread. Gynecol Oncol 1994;54:264-8.
12. Kato DT, Ferry JA, Goodman A, Sullinger J, Scully R, Goff B, et al. Uterine papillary serous carcinoma: a clinicopathologic study of 30 cases. Gynecol Oncol 1995;59:384-9.

13. Zheng W, Cao P, Zheng M, Kramer EE, Godwin TA. p53 overexpression and bcl-2 persistence in endometrial carcinoma: comparison of papillary serous and endometrioid subtypes. Gynecol Oncol 1996;61:167-74.

14. Inoue M, Fujita M, Enomoto T, Morimoto H, Monden T, Shimano T, et al. Immunohistochemical analysis of p53 in gynecologic tumors. Am J Clin Pathol 1994;102:665-70.

15. Manek S, Wells M. The significance of alterations in p53 expression in gynaecological neoplasms. Curr Opin Obstet Gynecol 1996;8:52-5.

16. Soong R, Knowles S, Williams KE, Hammond IG, Wysocki SJ, Iacopetta BJ. Overexpression of p53 protein is an independent prognostic indicator in human endometrial carcinoma. Br J Cancer 1996;74:562-7.

17. Tashiro H, Isacson C, Levine R, Kurman RJ, Cho KR, Hedrick L. p53 gene mutations are common in uterine serous carcinoma and occur early in their pathogenesis. Am J Pathol 1997;150:177-85.

18. Burton JL, Stewart RL, Heatley MK, Royds JA, Wells M. p53 expression, p21 expression and the apoptotic index in endometrioid endometrial adenocarcinoma. Histopathology 1999;35:221-9.

19. Lim P, Aquino-Parsons CF, Dupuis B, Phillips D, Zhou C, Gilks CB. Low-risk endometrial carcinoma: assessment of a treatment policy based on tumor ploidy and identification of additional prognostic indicators. Gynecol Oncol 1999;73:191-5.

20. Kohler MF, Carney P, Dodge R, Soper JT, Clarke-Pearson DL, Marks JR, et al. p53 overexpression in advanced-stage endometrial adenocarcinoma. Am J Obstet Gynecol 1996;175: $1246-52$.

21. Lim P, Al Kushi A, Gilks B, Wong F, Aquino-Parsons C. Early stage uterine papillary serous carcinoma of the endometrium: effect of adjuvant whole abdominal radiotherapy and pathologic parameters on outcome. Cancer 2001;91:752-7.

22. Hitchcock CL. Ki-67 staining as a means to simplify analysis of tumor cell proliferation. Am J Clin Pathol 1991;96:444-6.

23. Brown DC, Gatter KC. Monoclonal antibody Ki-67: its use in histopathology. Histopathology 1990;17:489-503.

24. Endl E, Gerdes J. The Ki-67 protein: fascinating forms and an unknown function. Exp Cell Res 2000;257:231-7.

25. Scholzen T, Gerdes J. The Ki-67 protein: from the known and the unknown. J Cell Physiol 2000;182:311-22.

26. Ambros RA. Simple hyperplasia of the endometrium: an evaluation of proliferative activity by Ki-67 immunostaining. Int J Gynecol Pathol 2000;19:206-11.

27. Lax SF, Pizer ES, Ronnett BM, Kurman RJ. Comparison of estrogen and progesterone receptor, Ki-67, and p53 immunoreactivity in uterine endometrioid carcinoma and endometrioid carcinoma with squamous, mucinous, secretory, and ciliated cell differentiation. Hum Pathol 1998;29:924-31.

28. Lax SF, Pizer ES, Ronnett BM, Kurman RJ. Clear cell carcinoma of the endometrium is characterized by a distinctive profile of p53, Ki-67, estrogen, and progesterone receptor expression. Hum Pathol 1998;29:551-8.

29. Kuwashima Y, Uehara T, Kishi K, Shiromizu K, Matsuzawa M, Takayama S. Proliferative and apoptotic status in endometrial adenocarcinoma. Int J Gynecol Pathol 1995;14:45-9.

30. Yaziji H, Gown AM. Immunohistochemical analysis of gynecologic tumors. Int J Gynecol Pathol 2001;20:64-78.

31. Duchrow M, Gerdes J, Schluter C. The proliferationassociated Ki-67 protein: definition in molecular terms. Cell Prolif 1994;27:235-42.

32. Yu CC, Filipe MI. Update on proliferation-associated antibodies applicable to formalin-fixed paraffin-embedded tis- 
sue and their clinical applications. Histochem J 1993;25:84353.

33. Elias LM. Cell proliferation indexes: a biomarker in solid tumors. Biotech Histochem 1997;72:78-85.

34. Yamauchi N, Sakamoto A, Uozaki H, Iihara K, Machinami R. Immunohistochemical analysis of endometrial adenocarcinoma for bcl-2 and p53 in relation to expression of sex steroid receptor and proliferative activity. Int J Gynecol Pathol 1996;15:202-8.

35. Salvesen HB, Iversen OE, Akslen LA. Prognostic significance of angiogenesis and Ki-67, p53, and p21 expression: a population-based endometrial carcinoma study. J Clin Oncol 1999;17:1382-90.

36. Geisler JP, Geisler HE, Miller GA, Wiemann MC, Zhou Z, Crabtree W. MIB-1 in endometrial carcinoma: prognostic significance with 5-year follow-up. Gynecol Oncol 1999;75:432-6.

37. Geisler JP, Wiemann MC, Zhou Z, Miller GA, Geisler HE. Proliferation index determined by MIB-1 and recurrence in endometrial cancer. Gynecol Oncol 1996;61:373-7.

38. Nordstrom B, Strang P, Bergstrom R, Nilsson S, Tribukait B. A comparison of proliferation markers and their prognostic value for women with endometrial carcinoma. Ki-67, proliferating cell nuclear antigen, and flow cytometric S-phase fraction. Cancer 1996;78:1942-51.

39. Silverberg SG. Histopathologic grading of ovarian carcinoma: a review and proposal. Int J Gynecol Pathol 2000;19:715

40. Mayr D, Diebold J. Grading of ovarian carcinomas. Int J Gynecol Pathol 2000;19:348-53.

41. Pirog EC, Czerwinski W. Diagnostic and prognostic significance of the mitotic index in endometrial adenocarcinoma. Gynecol Oncol 1992;46:337-40.

42. Tornos C, Silva EG, el-Naggar A, Burke TW. Aggressive stage I grade 1 endometrial carcinoma. Cancer 1992;70:790-8.

43. Announcements: FIGO stages-1988 revision. Gynecol Oncol 1989;35:125-7.

44. Ioffe OB, Papdimitrous JC, Drachenberg CB. Correlation of proliferation indices, apoptosis, and related oncogene expression (bcl-2 and c-erbB-2) and p53 in proliferative, hyperplastic, and malignant endometrium. Hum Pathol 1998; 29:1150-9.

\section{Book Review}

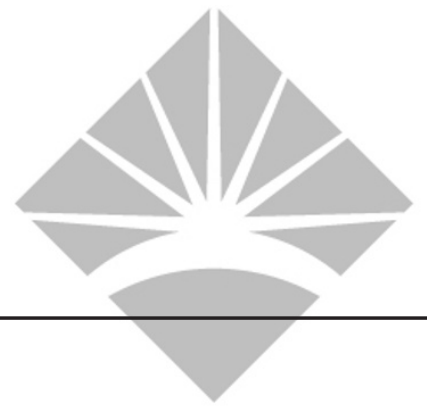

Wiernik P: Adult Leukemias, 300 pp, Hamilton, Ontario, Canada, 2001 (\$149.00).

The short text Adult Leukemias, by Peter Wiernik, M.D., is a well-written, comprehensive review of the clinicopathologic, immunophenotypic, cytogenetic, and therapeutic aspects of the leukemias. The book is written for clinicians, including hematologic/oncologists and pathologists, and students and residents of all levels of training. Dr. Wiernik and his distinguished list of contributors have made a complex topic quite understandable.

The authors have referenced important, recent papers from the literature. Many tables and charts are incorporated effectively throughout the book, particularly in the morphology and immunophenotyping chapters. In describing the leukemias, the authors reinforce the principles by using morphologic criteria, immunopheno- typic data, and cytogenetics together in several tables. The microscopic photographs are of excellent quality. Furthermore, the clinical photography and data covered in the chapter regarding extramedullary manifestations is very well done and diagnostically useful. There is some repetition of morphology and diagnostic classifications of the acute myeloid leukemias, as they are described in the text and various tables both in the diagnosis and the morphology chapters.

In summary, Dr. Wiernik has effectively achieved his goal of reviewing the advances in leukemia biology. The book is a good supplement and a nice addition to the hematologic literature.
John M. Oscherwitz
University of Kansas School of Medicine
Kansas City, Kansas 